\title{
Patterned Deposition of PS Gel is Controlled by Chitosan or Acetic Acid Additives
}

\author{
Yuji Kiyono and Olaf Karthaus* \\ Chitose Institute of Science and Technology, Bibi 65-758, Chitose 066-8655, Japan \\ (Received 8 September 2011; Accepted 21 September 2011; Published 9 November 2011)
}

\begin{abstract}
Phase-separation of polymers in thin films produces a wide variety of patterns. Here we show that a polystyrene/poly(methylmethacrylate solution can form stable phase separated structures when an additive such as chitosan or acetic acid is used. The phase separated films were analyzed by fluorescence microscopy. Possible applications of these films are as etching masks for solar cells or for security systems.
\end{abstract}

[DOI: $10.1380 /$ ejssnt.2011.404]

Keywords: Etching; Silicon; Surface roughness; Polymer; Phase separation; Nano structure chemistry; Processing and fabrication

\section{INTRODUCTION}

Nano- and microstructures of surfaces strongly influence the optical properties of the material, because of the modulation of the refractive index at such surfaces. Highly ordered photonic structures include photonic crystals-both natural such as opals as well as artificially produced such as wave guides. These highly periodic structures interact strongly with light and produce structural colors [1], or can be used as Bragg gratings [2] and so on.

Such regular structures can be prepared by top-down techniques such as photolithography, and by bottom-up such as self-assembly. Self-assembly is an elegant, low-cost process that allows for a parallel preparation of large-area devices, but with increasing processing speed, inaccuracies such as defects increase. For Bragg gratings, this results in lower quality.

For other applications in the field of photonics, the wavelength-dependent strong refraction of reflection of ligh is not necessary. Examples of low-order selforganzation in nature is the anti-reflection coating of moth eyes [3] and the 'whitest beetle' [4]. Thus, disordered structures on the submicrometer range can be sued for various optical and photonic applications, such as solar cells.

Solar cells do not require highly regular patterning of its surface. It has been reported that the reflection-loss of solar cells can be decreased by forming surface patterns on the semiconductor surface [5]. Thus, self-assembly of polymers is a very promising tool to improve the optical and photonic properties of the material.

Spinodal decomposition produces phase separated structures with a certain narrow wavelength $[6,7]$, but no in-plane order. Even less order is obtained when a polymer blend phase separates in a nucleation-and-growth mechanism.

Here we show that the phase separated structure that is formed when a solution of a mixture of two polymers, polytsyrene and poly(methylmethacryate), is cast on a substrate can be used as an etching mask for the surface roughening of a Si-substrate.

We could control the mechanism of the phase separation (spinodal vs. nucleation) by additives such as acetic

\footnotetext{
*Corresponding author: kart@photon.chitose.ac.jp
}

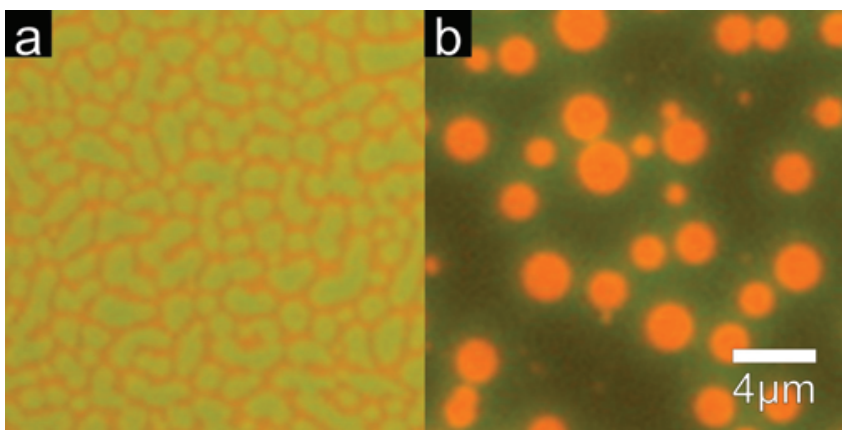

FIG. 1: Fluorescence micrographs of PS/PMMA blends cast from an ethyl acetate solution that contained 1\% chitosan and TCNQ as a fluorescence marker. The relative humidity was $40 \%$. The red parts correspond to PS, the green parts to PMMA.

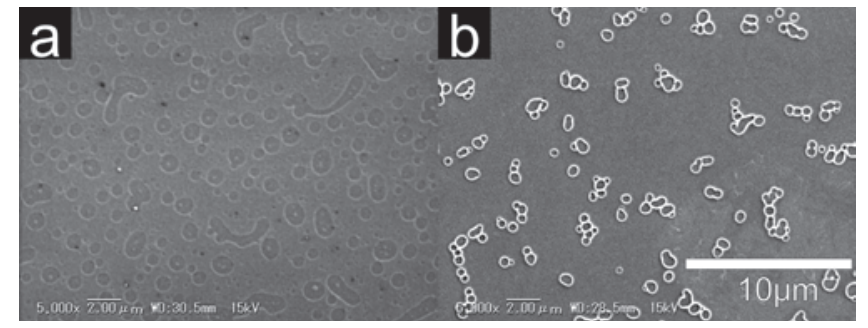

FIG. 2: Scanning electron micrographs of a PMMA pit structure on Si-wafer. After casting of the blend, the PS was dissolved by treatment with cyclohexane. A: chitosan as additive. B: acetic acid as additive. The scale bar is $2 \mu \mathrm{m}$.

acid or chitosan. Chitosan has been used as a coating for polystyrene microparticles [8], as well as in blends with PMMA to produce dentin cements [9]. The coating of the polystyrene particles is strong enough to survive the dissolution of the polystyrene core, leading to hollow chitosan spheres. The interaction of chitosan with PMMA is most likely through hydrogen bonding between the chitosan amino group with the polymeric ester groups. The details of the interaction with polystyrene are not known in detail, but the hydroxy- and amino groups of chitosan are all equatorial. There are no axial hydrophilic groups in chitosan and thus to a certain extent a hydrophobic interaction of polystyrene with chitosan is possible. By screening several biocompatible addictives, both low mlolar mass as well as polymeric, we found that the simple 


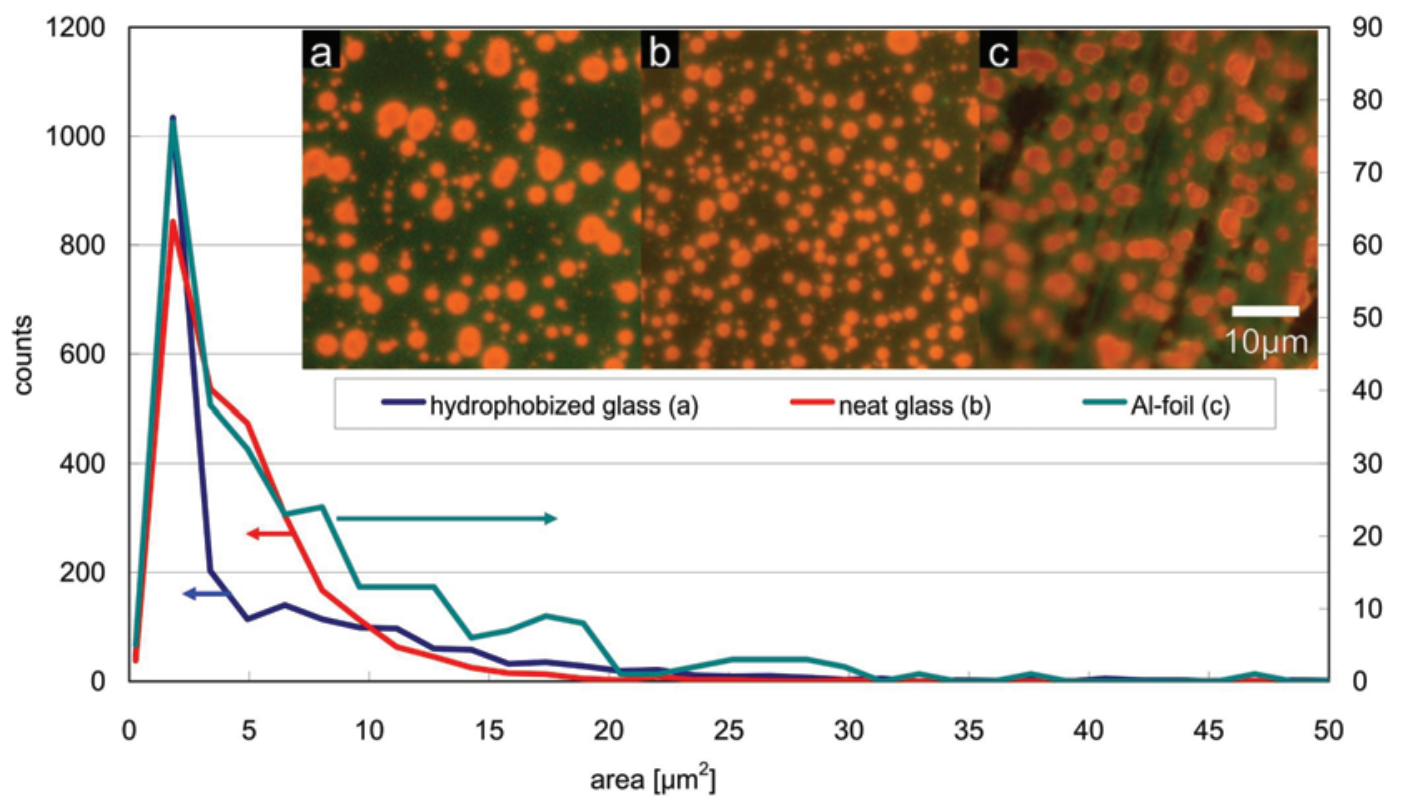

FIG. 3: Histogram of the size distribution of PS islands in a PS/PMMA blend film cast from an ethyl acetate solution that contained $1 \%$ chitosan and TCNQ as a fluorescence marker onto various substrates. The relative humidity during casting was $60 \%$. The surface area that was analyzed for the glass substrates was $250 \mu \mathrm{m} \times 180 \mu \mathrm{m}$, and for the Al substrate was $50 \mu \times$ $50 \mathrm{~mm}$. The inset shows fluorescence micrographs of three substrates. The red parts correspond to PS islands. a: hydrophobized glass, b: neat glass, c: Al-foil.
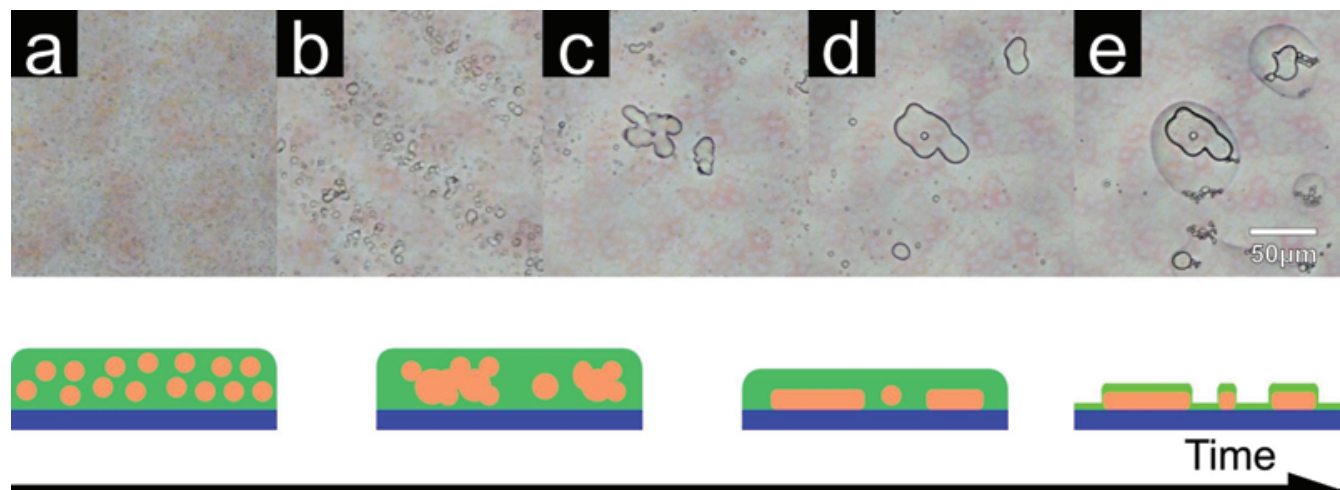

FIG. 4: Optical micrographs taken during the evaporation of a PS/PMMA blends cast from an ethyl acetate solution that contained $1 \%$ acetic acid. A: onset of the phase separation, $t=0 ; \mathrm{b}: t=14 \mathrm{~s} ; \mathrm{c}: t=25 \mathrm{~s} ; \mathrm{d}: t=36 \mathrm{~s} ; \mathrm{e}: t=51 \mathrm{~s}$. The scheme below the micrographs is an illustration of the phase separation of PS (orange) and PMMA (green). It should be noted that there is solvent present.

acetic acid plays a stabilizing role, too. Both chitosan and acetic acid are natural products that are biocompatible and biodegradable, thus reducing the strain on the environment for large scale production as compared to artificial additives.

As a result, polystyrene island structures with a diameter of $100 \mathrm{~nm}$ to $20 \mu \mathrm{m}$ have been achieved. These polystyrene islands can be selectively desorbed by treatment with cyclohexane. A dry etching process then leads to pits in the Si wafer that mirror the phase separated structure of the polymer blend.

Reflection measurements show that the etched patterned surfaces exhibits reduced reflectivity in the longwavelength region of the visible spectrum.

\section{EXPERIMENTAL PART}

\section{A. Materials}

The polymers used in this work were polystyrene (PS, Mw: 280,000, Aldrich), poly(methyl methacrylate) (PMMA, Mw: 120,000, Aldrich), Solvents for coating was ethyl acetate (AA, analytic grade, Wako pure chemical industries, ltd.). Additives for making pattern were acetic acid (Wako pure chemical industries, ltd.) and Chitosan (low molecular weight, Aldrich). Hydrochloric acid $(0.1 \mathrm{~mol} / \mathrm{l}$ analytic grade, Wako pure chemical industries, ltd.) was used to make a $3.0 \mathrm{mg} / \mathrm{mL}$ chitosan 
solution. The substrates used in this work were glass (18 mm $\times 18 \mathrm{~mm}$ and A4 size), Si wafer (KST world corp.), polyethylene (no additives Kitchen wrap) and aluminum (aluminum foil for food, TOYO ALUMINIUM EKCO PRODUCTS Co., Ltd). Polymer solution were prepared mixing the additive with the PS/PMMA or PS solution (typically $3 \mathrm{mg} / \mathrm{ml}$ of polymer by volume) for $10 \mathrm{~s}$ by a vortex mixer (IKA lab dancer). Polymer films were prepared by spin coating (MISAKA 1H-D7), casting and spraying (FURUPLA No. 3530). The polymer phases were identified by an added fluorescent dye, 1,4-Bis(dicyanomethylene)cyclohexadiene(TCNQ, TCI). This dye shows green fluorescence in the presence of PMMA and red fluorescence in the presence of PS [10].

\section{B. Pattern preparation}

Polymer films were prepared in dry nitrogen atmosphere, 20\%, 40-60\%, and 70\% humidity air. For imaging of the structures, a fluorescence microscope (Olympus BX-51), a scanning electron microscope (Keyence VE8800) and an AFM (JOEL JSPM-5200) was used. In situ video recording of the evaporating solution was obtained by a Nikon DIAPHOTO-300 inversted microscope that was placed in a sealed bag that allowed for controlling the atmospheric humidity. Dry etching was performed at the Research Institute for Electronic Science at Hokkaido University.

Reflectance of Si wafers was measured with a spectroscopic ellipsometer (JASCO M-150) between 400 and $800 \mathrm{~nm}$ at an angle of incidence of $45^{\circ}$.

\section{RESULTS}

Fluorescence microscopy shows that specific island structures were formed when a mixture of PS/PMMA containing either $1 \%$ of a chitosan solution or $0.5 \%$ acetic acid were cast at $40-60 \%$ humidity condition. Furthermore, pure polystyrene solutions that contained $1 \%$ of acetic acid solution gave polystyrene droplets on a substrate. Samples that contained no additives or samples that were cast under $20 \%$ humidity did not show the specific island pattern, but formed spinodal decomposition patterns, as can be seen in Fig. 1. Comparing the chitosan and acetic acid addive, it became clear that chitosan aides towards a more dense phase separated structure as can be seen in Fig. 2.

Figure 3 shows that the island structure is nearly independent of the substrate. All investigated substrates (glass substrates, Si wafer, polyethylene and aluminum foil) could be coated by our method. For the size distribution analysis, an surface area $250 \mu \mathrm{m} \times 180 \mu \mathrm{m}$ was analyzed. The thin aluminum foil showed bending on such a large area and parts of the image were out of focus. Thus, a smaller area that was flat $(50 \mu \mathrm{m} \times 50 \mu \mathrm{m})$ was used for the image analysis of the Al substrate. Figure 3 shows the histogram of the size distribution of the PS islands. The average size of the PS islands on neat glass is $1.11 \mu \mathrm{m}^{2}$ with a standard deviation (s.d.) of $0.44 \mu \mathrm{m}^{2}$; on hydrophobic glass it is $0.97 \mu \mathrm{m}^{2}$ (s.d. $0.42 \mu \mathrm{m}^{2}$ ), and

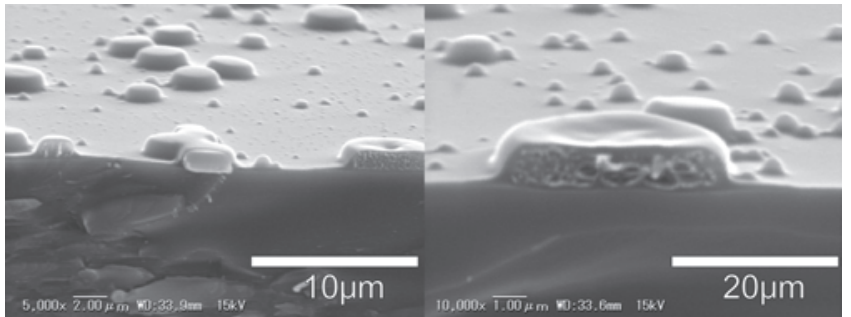

FIG. 5: Cross sectional scanning electron micrograph of a PS/PMMA blend cast from an ethyl acetate solution that contained $1 \%$ chitosan onto a glass slide. The right part is a blow-up of the picture on the left.

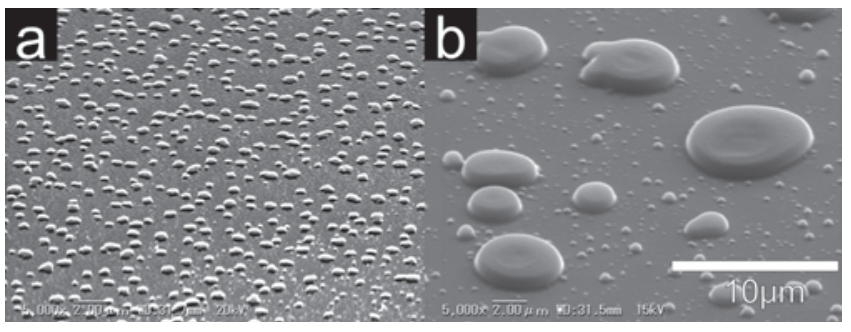

FIG. 6: SEM images of PS/PMMA blend ethyl acetate solution that contained $1 \%$ chitosan. A: a spin coated (500 rpm); B: solution cast.

on $\mathrm{Al}$ foil it is $1.23 \mu \mathrm{m}^{2}$ (s.d. $0.43 \mu \mathrm{m}^{2}$ ). Thus the histogram shows a peak at the same position. The hydrophobic glass contains nearly very few island $>5 \mu \mathrm{m}^{2}$, whereas the distribution on the neat glass is slightly broadened to $10 \mathrm{mum}^{2}$. The Al foil shows a few islands with sizes up to $20 \mu \mathrm{m}^{2}$. The insets show the fluorescence images of those three substrates. For clarity, a section of $50 \mu \mathrm{m} \times 50 \mu \mathrm{m}$ was chosen for all three substrates.

Not only the size distribution, but also the surface coverage of the PS islands does not vary much between those three substrates. From the images in Fig. 3 we calculated that it is $25 \%$ for neat glass, $23 \%$ for hydrophobic glass, and $24 \%$ for $\mathrm{Al}$ foil. Especially interesting is the fact that even the production-related high roughness of the aluminum foil (400 nm rms) had very little effect on the island structure.

In order to gain an insight into the formation mechanism, we observed the solvent evaporation in situ. From the formation process observed by optical microscopy (Fig. 4), it became clear that PS forms small droplets in the ethyl acetate solution (Fig. 4(a)). By convection, these droplets coalesce and form larger aggregates. The phase separated PS droplets contain enough solvent to be fluid enough so that the merging of droplets results in spherical aggregates (Fig. 4(b)). With progressing solvent evaporation, the viscosity of the solution increases and some of the larger coalescates cannot relax into spherical shapes anymore (Fig. 4(c)). They get fixed onto the substrate in irregular intervals (Figs. $4(\mathrm{~d})$ and (e)) that are nonetheless not random, due to the more or less regular convection pattern of the evaporating solution [11].

The homogeneous intensity of the fluorescence in Fig. 2 indicate that the island have a similar height. This was confirmed by SEM imaging (Fig. 5). The specific phase 

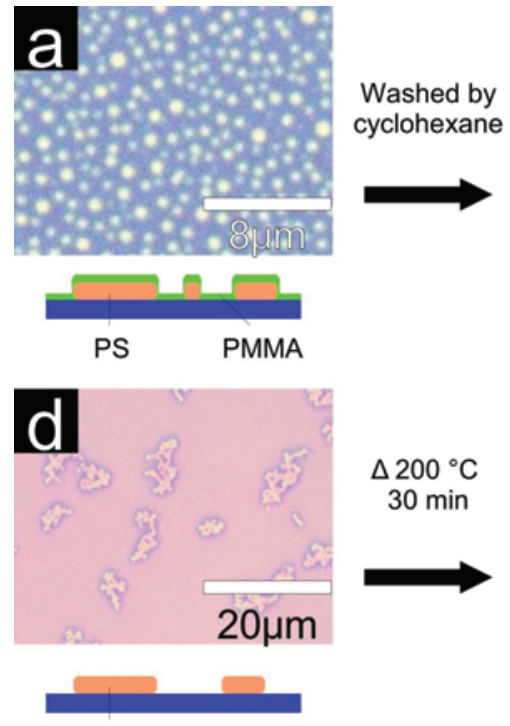

PS

\section{b}

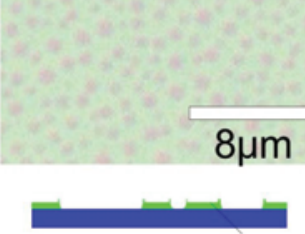

PMMA

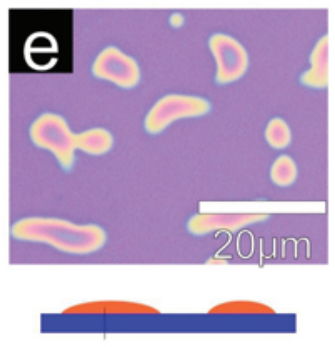

PS
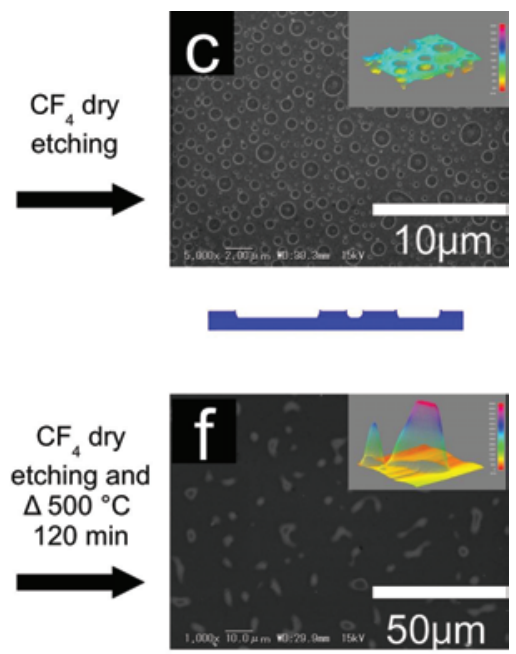

50 um

FIG. 7: Optical, SEM and AFM images illustrating the process of Si patterning. The upper row (a-c) shows the use of a PMMA porous mask, and the lower row (d-e) of a PS mask.
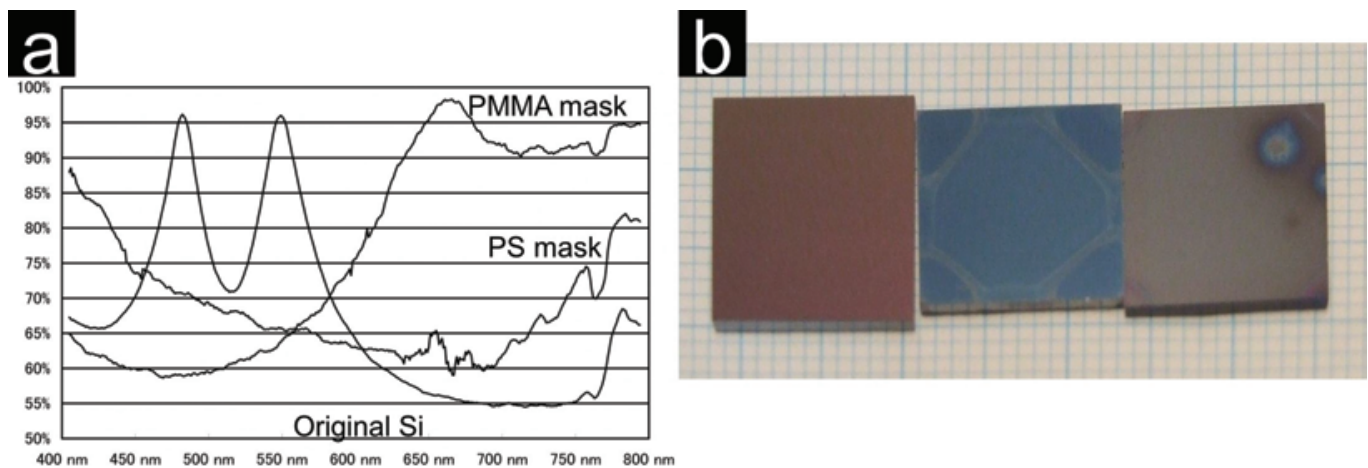

FIG. 8: A: Reflectance spectra of original Si wafer and after etching using PMMA and PS masks. The reflection was taken at an angle of $45^{\circ}$. B: photographs of white light reflected from wafers (left: original; center: after etching using a PMMA mask; right: after etching using a PS mask).

separation pattern of PS/PMMA contained islands with a height of $1 \mu \mathrm{m}$ that was more or less independent from the island diameter, which is called a 'mesa' structure. The mesa consist of a PS core covered with a thin PMMA skin that has a thickness of approx. $100 \mathrm{~nm}$. A cross section of the mesa shows an nanoscale internal void structure, most likely due to the presence of ethyl acetate in the PS gel that only evaporated after the mesa became solidified.

The mesa structure is formed while the gel is still deformable. The similar height comes from the thickness of the ethyl acetate layer just before it ruptures and the mesa height is fixed at that moment.

Since we could show that the size of the mesa grow my coalescence of small PS gel-like spheres, it should be possible to control the mesa size simply by changing the evaporation time of the ethyl acetate. Figure 6 is the proof that it is possible to control mesa sizes. The left picture (Fig. 6(a)) is a film obtained by spin coating a blend solution at $500 \mathrm{rpm}$, whereas the right picture (Fig. 6(b)) is taken from a sample that was solution cast. Spin coating prevents the coalescence of PS gel spheres and leads to a dense array of submicrometer-sized mesas.

\section{A. Resist mask formation and etching}

The mesa structure can be used as a resist mask. A PMMA resist mask was made from a film that was prepared by spin coating of a PS/PMMA solution with added $1 \%$ chitosan on Si wafer in $40 \%$ humid air. Then, this substrate was washed for $20 \mathrm{~min}$ with cyclohexane to remove PS [12] (Fig. 7), resulting in a porous film. SEM images show that the PMMA resist mask had a surface coverage of $66 \%$, and a pore diameter of about $500 \mathrm{~nm}$ (Fig. 7(b)). In addition, a PS resist mask made from a PS-only ethyl acetate solution with added acetic acid was also prepared (Fig. $7(\mathrm{~d})$ ). This resulted in the formation of PS islands on a bare Si substrate. The PS resist mask coverage was $17 \%$, the area of a single PS island was about $30 \mu \mathrm{m}^{2}$.

The PS patterns was heated to $200^{\circ} \mathrm{C}$ for $30 \mathrm{~min}$ to 


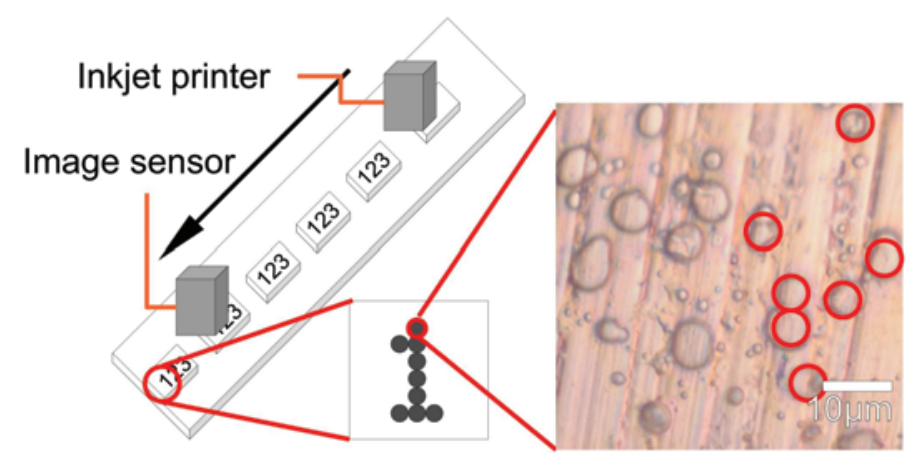

FIG. 9: Schematic representation of printing a phase separated polymer structure using an ink-jet printer. The phase separated structure can be imaged by a low resolution microscope. An image processing software can be used to automatically recognize the phase separated PS mesa (as indicated by the red circles in the right part of the photograph).

increase adhesion of the polymer to the substrate and to condense the PS into islands without voids (Fig. 7(e)). Then, the Si wafers were dry etched (SAMCO RIE-10NR) with CF4. The depth of the etched structures holes in the case of the PMMA mask was $30 \mathrm{~nm}$, and the height of the islands in the case of the PS mask was $500 \mathrm{~nm}$, as can be seen in Fig. 7.

The reflectance measurements using an ellipsometer show that the reflectivity of the etched $\mathrm{Si}$ wafers is lower than the one of planar wafers (Fig. 8). The color of the wafer changes from purple (as received) to blue (PMMA mask) or gray (PS mask) indicating that these etched structures may be useful to produce structures that should show a similar effect as silicon microwires reported in the literature $[13,14]$ for the preparation of solar cells.

\section{B. Security system}

Figure 9 explains how the mesa pattern may be useful for anti-counterfeit technology (disposable authentication) because of the complex and irreproducible microscopic pattern is difficult to fake, but on the other hand very easy to confirm by a low resolution optical microscopy. The blend polymer solution can be printed by an inkjet printer (for example when printing the expiration date on a milk carton). As we have shown, the pat- tern can be produced on many different substrates and in ambient temperature and environment. The individual pattern in one of the inkjet spots can be images, digitalized and then used to generate a code that links it to the macroscopic inkjet characters.

A security system then can record the pattern by a simple USB microscope and match the coded pattern to the characters.

\section{SUMMARY}

In this paper we have shown that a solution of ethyl acetate, an environmentally friendly solvent, can be used to prepare micronsized phase separated structures in a PS/PMMA polymer blend. The patterns can be used as etching masks for the preparation of low-reflection Si wafers and as a counterfeit-proof encoding using ink-jet printer.

\section{Acknowledgments}

We thank Dr. Matsuo at the Research Institute for Electronic Science, Hokkaido University for his help in etching the Si wafers.
[1] T. F. Anderson and A. G. Richards, J. Appl. Phys. 13 748 (1942)

[2] T. F. Krauss, R. M. DeLaRue, and S. Brand, Nature 383 699 (1996).

[3] T. Yanagishita, K. Yasui, T. Kondo, Y. Kawamoto, K. Nishio, and H. Masuda, Chem. Lett. 36, 530 (2007).

[4] M. J. Lander, Biophotonics International 14, 84 (2007).

[5] T. A. Gessert and T. J. Coutts, J. Vac. Sci. Technol. 10, 2013 (1992).

[6] S. Walheim, M. Boltau, J. Mlynek, G. Krausch, and U. Steiner, Macromolecules 30, 4995 (1997).

[7] S. Y. Heriot and R. A. L. Jones, Nat. Mater. 4, 782 (2005).

[8] Z. Qian, Z. Zhang, H. Li, H. Liu, and Z. Hu, J. Polym. Sci. A: Polym. Chem. 46, 228 (2008).

[9] N. Flores-Ramírez, G. Luna-Bárcenas, S. R. Vásquez-
García, J. Muñoz-Saldaña, E. A. Elizalde-Peña, R. B. Gupta, I. C. Sanchez, J. González-Hernández, B. GarciaGaitan and F. Villasenor-Ortega, J. Biomat. Sci. Polym. Ed. 19, 259 (2008).

[10] O. Karthaus and Y. Kiyono, e-J. Surf. Sci. Nanotech. 4, 270 (2006).

[11] O. Karthaus, L. Grasjo, N. Maruyama, and M. Shimomura, Chaos 9, 308 (1999).

[12] C. Deng, C. Xu, L. Xu, D. Zou, W. Jiang, T. Dai, X. Li, and G. Sheni, Polym. Mater. Sci. Engin. 1, 151 (2010).

[13] K. Park, Z. Guo, H. Um, J. Jung, J. Yang, S. Lim, Y. Kim, and J. Lee, Optics Express 19, A41 (2011).

[14] M. Park, K. Park, Z. Guo, J. Jung, H. Um, Y. Nam, S. Shin, and J. Lee, 5th International Conference on Nanophotonics 2011, Shanghai, p. 53 (2011). 